\title{
The Brief Case
}

(For answers to the self-assessment questions and take-home points, see page 2842 in this issue [doi:10.1128/JCM.01022 -16].)

\section{Probable Transfusion-Transmitted Babesiosis in a Transplant Recipient}

\author{
Eimear Kitt, ${ }^{a}$ Amelia A. Keaton, ${ }^{a}$ Erin H. Graf ${ }^{\text {b,c }}$ \\ Division of Infectious Diseases, Children's Hospital of Philadelphia, Philadelphia, Pennsylvania, USA a Department of Pathology and Laboratory Medicine, Children's \\ Hospital of Philadelphia, Philadelphia, Pennsylvania, USA ; Department of Pathology and Laboratory Medicine, Perelman School of Medicine, University of Pennsylvania, \\ Philadelphia, Pennsylvania, USA
}

\section{CASE}

3-year-old immunocompromised male who had been hospitalized for 7 months in the cardiac intensive care unit developed fever and tachycardia in December. He was prenatally diagnosed with hypoplastic left heart syndrome and received right ventricle to pulmonary artery conduit surgery days after delivery. Due to worsening right ventricular function, after a hemi-Fontan procedure, he received an orthotopic heart transplant at the beginning of his 3 rd year of life. His posttransplant course was complicated by multiple episodes of rejection, cytomegalovirus (CMV) pneumonitis, and several central-line-associated bloodstream infections. As a result of the rejection, necessitating plasmapheresis as well as frequent blood draws for management, which led to anemia, he received 36 packed red blood cell transfusions over the course of 7 months posttransplantation. These transfusions were evenly spaced, and he remained hospitalized during the 7 months. At the time of the febrile episode, he was on caspofungin, trimethoprim-sulfamethoxazole, and ganciclovir. The subsequent diagnostic workup included multiple sets of blood cultures, a urine culture, and a CMV viral load analysis. $\mathrm{He}$ was started on vancomycin and cefepime while awaiting microbiologic results. Other pertinent test results included a complete blood count (CBC) with differential showing pancytopenia and an aspartate transaminase (AST) level of $200 \mathrm{U} /$ liter (reference range, 20 to $60 \mathrm{U} /$ liter), an alanine aminotransferase (ALT) level of $84 \mathrm{U} /$ liter (reference range, 5 to $45 \mathrm{U} /$ liter), and a C-reactive protein level of $3.1 \mathrm{mg} / \mathrm{dl}$ (reference range, 0 to $0.9 \mathrm{mg} / \mathrm{dl}$ ), which increased to $7.1 \mathrm{mg} / \mathrm{dl}$ over 4 days. On the 5 th day of fever, another CBC with differential was ordered and was noted by the hematopathologist to contain intraerythrocytic parasites. Immediately, a blood smear with Giemsa stain (Harleco Giemsa stain; EMD Millipore, Billerica, MA, USA) was performed by the microbiology laboratory, yielding the definitive diagnosis (Fig. 1). Babesia species with a parasitemia level of $18 \%$ was reported to the clinical team. Real-time PCR testing, performed by a reference laboratory, provided the species-level identity of Babesia microti. All other infectious workups were negative. The patient was started on azithromycin plus atovaquone due to the contraindications against treatment with quinidine (QT interval prolongation in a heart transplant recipient with declining heart function). Three days later, clindamycin was added when his parasitemia level did not decline. He was also given an exchange transfusion on day 4 after diagnosis in an attempt to reduce his parasitemia. After 14 days of therapy, his parasitemia became undetectable and he completed 6 full weeks of therapy, at which time he remained aparasitemic
(Fig. 2). Since transfusion was the child's only known risk factor for Babesia infection, a complete investigation into the blood products used was conducted, but the infectious unit/donor could not be definitively identified. Banked products from the organ donor were also tested, and it was determined that the heart transplant was not the source of the Babesia infection.

\section{DISCUSSION}

Babesia species are members of the protozoal phylum Apicomplexa and as such are distantly related to Plasmodium species, the causative agent of malaria. As with Plasmodium spp., transmission occurs via vector and replication occurs inside human erythrocytes. There are at least 7 species of Babesia described to cause human diseases found throughout the world; however, the phylogeny is still in flux, and new species are likely to be defined (1). In the United States, B. microti is by far the dominant species associated with human infections (2). Infections due to all other species of Babesia are extremely rare; there have been a few cases of Babesia duncani and B. duncani-like species reported in the Pacific coastal region and three cases of a Babesia divergens-like species, two in the midwestern United States, and one in Washington State (1). Outside of the United States, cases of babesiosis due to any species (including B. microti) are infrequent, with several dozen cases of $B$. divergens reported throughout Europe and similar numbers of cases of Babesia venatorum reported in China $(1,2)$.

The primary vector for all Babesia species is the hard-bodied tick, Ixodes spp. It is the nymph stage in the Ixodes life cycle that is responsible for transmission due to its small size (unlikely to be recognized and removed) and questing behavior, whereby it perches at the top of foliage, extending its legs in order to attach to vertebrates passing by. In the United States, B. microti is vectored by the Ixodes scapularis tick. This species of tick also happens to be the vector for the causative agent of Lyme disease, the bacterium Borrelia burgdorferi, as well as for the causative agent of human granulocytic anaplasmosis, the bacterium Anaplasma phagocytophilum. Since babesiosis became a nationally notifiable condition in 2011, it is not surprising that almost all reported cases overlap

Citation Kitt E, Keaton AA, Graf EH. 2016. Probable transfusion-transmitted

babesiosis in a transplant recipient. J Clin Microbiol 54:2632-2634. doi:10.1128/JCM.00981-16

Editor: C.-A. D. Burnham, Washington University School of Medicine

Address correspondence to Erin H. Graf, grafe@email.chop.edu.

Copyright @ 2016, American Society for Microbiology. All Rights Reserved. 


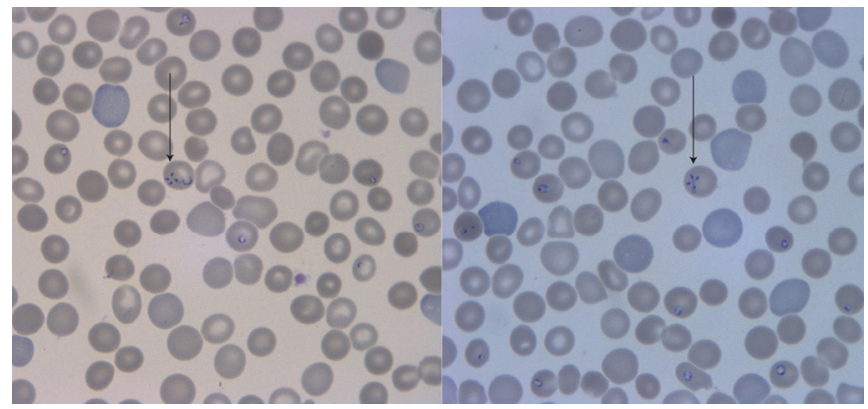

FIG 1 Giemsa-stained thin blood smears. Trophozoite or ring forms are visible inside the erythrocytes. The arrows point to tetrads, often referred to as Maltese crosses, which are the result of asexual budding of trophozoites.

the geographic distribution and seasonal incidence of Lyme disease, and coinfections have been reported. More than 95\% of roughly 1,700 cases reported each year come from northeastern and upper-midwestern states, with most cases being reported from June to August, i.e., during periods of nymphal tick and human convergence (3).

The clinical presentation of babesiosis is quite variable, and its severity depends largely on the host. The estimated incubation period from tick bite to development of symptoms ranges from 1 to 9 weeks $(1,2)$; however, most infected individuals do not recall a tick bite. The most common clinical presentations include nonspecific symptoms, such as fever, headache, chills, malaise, arthralgia, and/or nausea, which may complicate diagnosis due to the overlap with other more common infections. The most-severe manifestations are seen in elderly, immunocompromised, and asplenic individuals, who also have a high parasitemia and mortality rate associated with infection $(1,2)$. Importantly, asymptomatic infection is perhaps more common than previously appreciated, as studies have documented wide discordance between seroprevalence and reported cases in areas of endemicity (4).

The gold standard for diagnostic testing is examination of Giemsa-stained thick and thin blood smears, the same method used for diagnosis, species-level identification, and parasitemia calculation for Plasmodium spp. Trophozoite forms of Babesia spp. are readily visualized inside erythrocytes as "ring" shapes (see Fig. 1 for examples). The morphology can easily be confused with Plasmodium falciparum, which can also form small ring-shaped trophozoites. The major morphological difference between these organisms is the presence of frequent extracellular Babesia trophozoites as well as Babesia "tetrads," or four connected trophozoites (often referred to as a Maltese cross). These distinctive forms are not always visible, particularly in cases of low parasite burden. In the case presented here, only the tetrad forms were visible (Fig. 1, arrows), as there were no extracellular forms despite a very high parasitemia level. Amplification and detection of Babesia DNA are a more sensitive and specific method than blood smear examination, and species-level identifications vary by assay design; however, testing is limited to reference laboratories, as no commercial or FDA-cleared methods exist. Furthermore, molecular testing does not provide quantitative information, which can be critical for treatment monitoring. Like molecular testing, assays for IgG and IgM antibodies are available only through reference laboratories and may be useful in very specific circumstances, such as in blood donor examination, in cases of chronic infection with

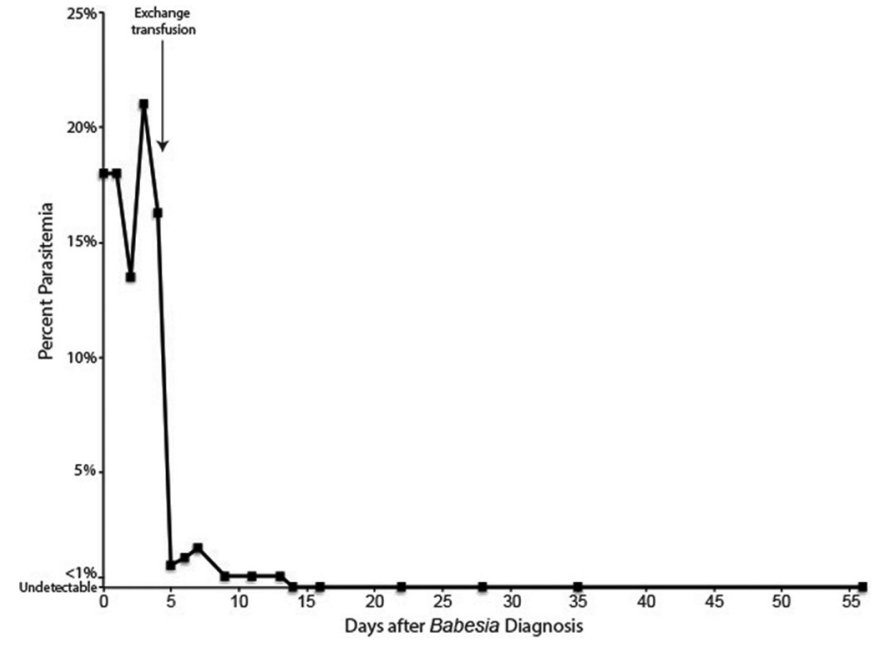

FIG 2 Serial monitoring of percent parasitemia. The percent parasitemia is shown for each blood sample collected after the initial diagnosis on day 0 . An exchange transfusion was performed on the 4 th day after the initial diagnosis. The parasitemia dropped below $1 \%$ on the 9 th day after the initial diagnosis, and parasites were no longer visible on Giemsa-stained smears 2 weeks after the initial diagnosis and beyond.

undetectable parasitemia, or for species-level identification purposes, as antibodies are highly species specific. A single IgG immunofluorescence antibody (IFA) titer of greater than or equal to $1: 256$ is considered supportive evidence of infection in the CDC case definition, but molecular or blood smear detection is required for confirmation.

Recommended treatment regimens and durations depend upon the severity of babesiosis. Typically, 7 to 10 days of either atovaquone plus azithromycin or clindamycin plus quinine is the frontline course, with the latter combination (clindamycin administered intravenously) preferred for severe cases. Longer duration of therapy and/or exchange transfusions may be indicated in cases of double-digit parasitemia levels or cases in which parasitemia is refractory to treatment $(1,2)$.

Transfusion-transmitted babesiosis (TTB) is a well-described phenomenon that has increased in frequency over the last 2 decades $(2,5)$. While babesiosis is the leading cause of transfusiontransmitted infection and transfusion-transmitted infection-related death (2), it is not currently screened for by laboratory testing of the U.S. blood supply. Screening for Babesia infection is performed only by questioning potential donors about a history of babesiosis and their current health status and a predonation evaluation for anemia. In a comprehensive review of 159 transfusiontransmitted babesiosis cases, most transmissions occurred in states where babesiosis is endemic but without a seasonal pattern (5). In addition, the time from symptom onset to diagnosis was a median of 6 days (range, 0 to 54 days), reflecting the fact that babesiosis is not likely to be considered in the differential due to a presumed lack of risk factors. In fact, diagnosis in these cases was often incidental due to visualization of the parasite in a manual differential. All of these features are similar to those of the case presented here. This patient acquired B. microti in a state where the disease is endemic. The patient became symptomatic in the middle of winter, and 5 days elapsed from presentation to diagnosis. The parasitic infection was identified only incidentally on a $\mathrm{CBC}$ with a manual differential. Another important piece of data 
from this same comprehensive review is the median time from transfusion to symptom onset of 37 days (with a range from 11 to 176 days) (5). This has important implications for donor tracing, as many transfusion recipients receive multiple units across months from different donors. In the case presented here, 36 units were received within the high end of the reported range (176 days), leading to a complicated investigation.

Several blood supply screening strategies have been proposed, and models of cost-effectiveness have been reported (6). Most reports agree that targeted screening for $B$. microti, focused on blood collected in areas of endemicity, is the best approach. Both antibody and molecular testing approaches have been proposed, together or separately. Antibody screening has been implicated as the most cost-effective and likely the most sensitive approach due to the persistence of antibodies in chronically infected persons. However, current methodology (indirect immunofluorescence assay) is neither FDA cleared nor high throughput. Molecular testing may be cost-effective only as an adjunct to serologic testing during periods of high Babesia transmission (i.e., summer) to detect acute preseroconversion infections. As an alternative to screening, universal pathogen reduction technologies, such as induced nucleic acid damage, have shown promise by inactivating Babesia species in blood products. These methods have yet to receive FDA approval for whole blood but have recently been approved for plasma and platelet treatment (2).

In summary, babesiosis is an emerging infection that is of increasing importance due to its presence in the blood supply and lack of approved methods for laboratory screening. Diagnosis is complicated by a high frequency of asymptomatic carriage, nonspecific symptoms, and lack of consideration of babesiosis in the differential for transfusion recipients.

\section{SELF-ASSESSMENT QUESTIONS}

1. What is the median incubation period for transfusiontransmitted babesiosis (time from transfusion to symptoms)?
(a) 7 days
(b) 18 days
(c) 37 days

(d) 83 days

2. What is the primary vector for Babesia in the United States, and what other pathogen can it transmit?
(a) Ixodes scapularis, Borrelia burgdorferi
(b) Dermacentor variabilis, Rickettsia rickettsii
(c) Amblyomma americanum, Ehrlichia chaffeensis
(d) Ornithodoros hermsi, Borrelia recurrentis

3. What is the gold standard test method for definitive diagnosis of Babesia infection?

(a) Hamster inoculation

(b) Real-time PCR

(c) Giemsa-stained blood smear

(d) IgG/IgM antibody detection by indirect immunofluorescence

\section{REFERENCES}

1. Vannier EG, Diuk-Wasser MA, Ben Mamoun C, Krause PJ. 2015. Babesiosis. Infect Dis Clin North Am 29:357-370. http://dx.doi.org/10.1016/j .idc.2015.02.008.

2. Leiby DA. 2011. Transfusion-transmitted Babesia spp.: bull's-eye on Babesia microti. Clin Microbiol Rev 24:14-28. http://dx.doi.org/10.1128/CMR $.00022-10$.

3. Adams D, Fullerton K, Jajosky R, Sharp P, Onweh D, Schley A, Anderson W, Faulkner A, Kugeler K. 2015. Summary of notifiable infectious diseases and conditions-United States, 2013. MMWR Morb Mortal Wkly Rep 62:1-122. http://dx.doi.org/10.15585/mmwr.mm6253a1.

4. Johnson ST, Cable RG, Tonnetti L, Spencer B, Rios J, Leiby DA. 2009. Seroprevalence of Babesia microti in blood donors from Babesia-endemic areas of the northeastern United States: 2000 through 2007. Transfusion 49:2574-2582. http://dx.doi.org/10.1111/j.1537-2995.2009.02430.x.

5. Herwaldt BL, Linden JV, Bosserman E, Young C, Olkowska D, Wilson M. 2011. Transfusion-associated babesiosis in the United States: a description of cases. Ann Intern Med 155:509-519. http://dx.doi.org/10.7326 10003-4819-155-8-201110180-00362.

6. Bish EK, Moritz ED, El-Amine H, Bish DR, Stramer SL. 2015. Costeffectiveness of Babesia microti antibody and nucleic acid blood donation screening using results from prospective investigational studies. Transfusion 55:2256-2271. http://dx.doi.org/10.1111/trf.13136. 